CARDIOVASCULAR MEDICINE

\title{
Increased titres of anti-human heat shock protein 60 predict an adverse one year prognosis in patients with acute cardiac chest pain
}

\author{
D H Birnie, L E Vickers, W S Hillis, J Norrie, S M Cobbe
}

Heart 2005;91:1148-1153. doi: 10.1136/hrt.2004.040485

See end of article for authors' affiliations .....................

Correspondence to: Dr David Birnie, Room H1 45, Ottawa Heart Institute, 40 Ruskin Road Ottawa, Ontario, Canada K1Y 4W7; dbirnie@ oftawaheart.ca

Accepted 18 January 2005
Objective: To assess whether antibodies to human heat shock protein 60 (anti-huhsp60) or to mycobacterial heat shock protein 65 (anti-mhsp65) predict an adverse one year prognosis in patients admitted with acute cardiac chest pain.

Design: Prospective observational study.

Setting: Teaching hospital.

Patients: 588 consecutive emergency admissions of patients with acute chest pain of suspected cardiac origin.

Main outcome measures: Anti-huhsp60 and anti-mhsp65 titres were assayed on samples drawn on the morning after admission. The end points after discharge were coronary heart disease death, non-fatal myocardial infarction, coronary artery bypass grafting, percutaneous transluminal coronary angioplasty, angiogram, or readmission with further cardiac ischaemic chest pain.

Results: During follow up after discharge (mean of 304 days, range 1-788 days), 277 patients had at least one of the study outcomes. Patients with increased titres of anti-huhsp60 had an adverse prognosis (hazard ratio 1.56 (95\% confidence interval 1.09 to 2.23 ) comparing highest versus lowest quartiles, $\mathrm{p}=0.015)$. Anti-mhsp65 titres were not predictive.

Conclusions: Patients admitted with acute cardiac chest pain and increased titres of anti-huhsp60 had an adverse one year prognosis.
$\mathrm{T}$ he inflammatory state of atherosclerosis is important in determining plaque stability. Increased $C$ reactive protein (CRP) concentrations have important prognostic implications and interest has turned to other aspects of inflammation including antibodies to heat shock protein (hsp) 60/ 65. The hypothesis linking hsp60/65 with atherosclerosis originated from the work of $\mathrm{Xu}$ and colleagues. ${ }^{1-6}$

Xu et al ${ }^{2}$ published in 1993 the first clinical study relating autoimmunity to hsp60/65 with atherosclerosis. They showed that an increased antimycobacterial hsp65 (anti-mhsp65) titre was independently associated with the presence of carotid atherosclerosis. ${ }^{2}$ Subsequently some studies have found a positive association between anti-msp $65^{78}$ and anti-human hsp60 (anti-huhsp60) (-11 $^{-1}$ tres and prevalent coronary atherosclerosis. Four large prospective studies ${ }^{12-15}$ have shown significant associations of anti-huhsp60 or antimhsp65 titre with development of carotid atherosclerosis ${ }^{13}$ or with clinical events. ${ }^{12} 1415$

The patients in these prospective studies were all stable at inclusion. This study is the first to assess whether increased titres of anti-huhsp60 have prognostic significance in patients presenting with unstable coronary heart disease (CHD), where the atherosclerotic inflammatory state may be importantly different from the stable baseline state.

\section{METHODS}

\section{Patients}

All patients admitted to the emergency department with acute chest pain of suspected cardiac ischaemic origin were eligible for inclusion. Patients were assessed on the first morning after admission to hospital by patient history, clinical examination, and available 12 lead ECGs. ECG abnormalities or other evidence of coronary artery disease was not required for enrolment. The only exclusion criteria were non-ischaemic chest pain; definite ST elevation acute myocardial infarction (MI); poor one year non-cardiac prognosis; and lack of informed consent. All patient management decisions were made solely by the treating physician. The protocol was approved by the hospital's ethics committee.

\section{Clinical assessment}

All patients were seen by a single investigator ( $\mathrm{E} \mathrm{V} \mathrm{V),} \mathrm{who}$ obtained written informed consent from all patients. A standardised history and physical examination were performed and the results were entered on to case record forms.

\section{Electrocardiography}

One of the investigators (LEV) evaluated the admission ECG. The ECG was categorised as follows: (a) previous myocardial infarction (MI, pathological Q waves, an otherwise unexplained positive $\mathrm{R}$ wave in lead $\mathrm{Vl}$ indicative of established posterior MI, or left bundle branch block); (b) significant ST$\mathrm{T}$ wave abnormality ( $\geqslant 1 \mathrm{~mm}$ ST segment deviation in two or more contiguous leads, or $\geqslant 1 \mathrm{~mm} \mathrm{~T}$ wave inversion in two or more contiguous leads with predominantly positive QRS complexes; no previous MI); (c) minor abnormality (any ECG abnormality not satisfying either of the above criteria; no previous MI or significant ST-T wave abnormality); and (d) normal. All subsequent ECGs recorded during the admission stay were analysed for evidence of new MI and significant

Abbreviations: $B S A$, bovine serum albumin; $C A B G$, coronary artery bypass grafting; $\mathrm{CHD}$, coronary heart disease; $\mathrm{Cl}$, confidence interval; CRP, C reactive protein; ELISA, enzyme linked immunosorbent assay; hsp, heat shock protein; huhsp, human heat shock protein; mhsp, mycobacterial heat shock protein; MI, myocardial infarction; PBS, phosphate buffered saline; PTCA, percutaneous transluminal coronary angioplasty 
new ST-T wave changes as defined by category (b) above when compared with the most recent recording.

\section{Assays}

On the first morning after admission, $20 \mathrm{ml}$ of venous blood was obtained. The blood was centrifuged for 30 minutes at $2000 \mathrm{~g}$ and $4^{\circ} \mathrm{C}$. Plasma was collected and stored at $-70^{\circ} \mathrm{C}$ until use. Anti-mhsp65 and anti-huhsp60 titres were subsequently measured. The first of these assays is an enzyme linked immunosorbent assay (ELISA) based on a modification of the method of $\mathrm{Xu}$ et $\mathrm{al}^{16}$ and we have previously validated and published the technique. ${ }^{7}{ }^{17}$ Briefly, microtitre plates were coated with $1 \mu \mathrm{g} / \mathrm{ml}$ of recombinant mhsp65 in $100 \mu \mathrm{l}$ phosphate buffered saline (PBS) in each well at $4^{\circ} \mathrm{C}$ overnight. The plates were then washed with a $0-01 \%$ Tween solution in PBS and blocked with $200 \mu \mathrm{l} 0-1 \%$ bovine serum albumin (BSA) in PBS (PBS-BSA) at room temperature for one hour. The plates were washed again and then incubated with $100 \mu \mathrm{l}$ of serum samples diluted 1:400 with PBS-BSA. After a further wash in PBS-Tween, the plates were incubated with horseradish peroxidase conjugated rabbit anti-human IgG (Dakopatts, Glostrup, Denmark) diluted 1:3000 with PBS-BSA. This was left at room temperature for one hour and the plates were washed with PBS-Tween again. Colour was developed with $o$-phenylenediamine and the reaction was stopped with $4 \mathrm{~mol} / \mathrm{H}_{2} \mathrm{SO}_{4}$. The standard consisted of caprylic acid purified IgG from a patient with a high anti-mhsp65 concentration. The mean absorbance was calculated for each test sample and the serial dilutions of the standard. The unknown values for each test sample were read against the standard curve and values expressed as U/l. The anti-huhsp60 assay was developed along similar lines. Recombinant huhsp60 was obtained form StressGen Biotechnologies Corp (Victoria, British Columbia, Canada).

CRP was assayed by an in-house ELISA with rabbit antihuman CRP antibodies on Nunc microtitre plates. The sensitivity of this assay is $0.001 \mathrm{mg} / \mathrm{l}$. Troponin $\mathrm{T}$ was determined by a chemiluminescent sandwich immunoassay and the sensitivity of this assay was $0.01 \mu \mathrm{g} / \mathrm{l}$. Physicians were blinded to the results all assays.

\section{Follow up}

After discharge, the patients were followed up by review of their hospital case records and directly by telephone or letter. General practitioners were contacted by letter when direct patient contact was unsuccessful. Case records from other hospitals or primary care were reviewed in cases where patients had possible end points in other hospitals or the community.

\section{End point}

The hard end point was defined as time to the first of death caused by CHD, including sudden death, or non-fatal MI. The hard plus soft end point was time to the first of CHD death, non-fatal MI, coronary artery bypass grafting (CABG), percutaneous transluminal coronary angioplasty (PTCA), angiogram, or readmission to hospital with chest pain. Angiograms, CABGs, and PTCAs already arranged before the index admission were not counted as end points. Angiograms performed or arranged during the index admission also were not counted.

\section{Statistical analysis}

The characteristics of the cohort were summarised by the number (\%) of patients for categorical covariates and mean (SD) or median (interquartile range) for continuous covariates (table 1 lists the covariates of interest). Anti-huhsp60, anti-mhsp65, and CRP were log transformed for analysis. Spearman rank correlations were calculated between the

\section{Table 1 Baseline characteristics}

\begin{tabular}{ll}
\hline Categorical covariates & No (\%) \\
Male sex & $312(53.2 \%)$ \\
Previous stroke & $52(8.9 \%)$ \\
History of hypertension & $255(43.4 \%)$ \\
History of hyperlipidaemia & $184(31.3 \%)$ \\
Diabetes & $86(14.6 \%)$ \\
Current smoking & $224(38.1 \%)$ \\
History of CHD* & $283(48.1 \%)$ \\
Aspirin & $320(54.6 \%)$ \\
$\beta$ Blocker & $129(22.1 \%)$ \\
ACE inhibitor & $101(17.3 \%)$ \\
Lipid lowering treatment & $51(8.7 \%)$ \\
Admission ECG & \\
Normal & $80(13.6 \%)$ \\
Minor abnormality & $233(39.6 \%)$ \\
Significant ST-T abnormality & $158(26.9 \%)$ \\
Previous MI & $117(19.9 \%)$ \\
New ECG changes during index admission & $110(18.7 \%)$ \\
Continuous covariates & Mean (SD) \\
Age (years) & $62.4(11.9)$ \\
White cell count (10 9 /I) & $9.1(3.3)$ \\
Loge (sensitive CRP) (loge mg/l) & $1.71(1.36)$ \\
Creatinine (IU/I) & $99(36)$ \\
Loge (anti-huhsp60) (U/I) & $3.25(0.71)$ \\
Loge (anti-mhsp65) (U/I) & $2.81(0.95)$ \\
\hline *Defined as any previous documented myocardial infarction (MI), \\
previous coronary artery bypass grafting (CABG), previous percutaneous \\
transluminal coronary angioplasty (PTCA), significant coronary heart \\
disease (CHD; $\geqslant 50 \%$ stenosis) on previous angiogram, previous positive \\
stress test (treadmill or thallium), or ECG evidence of previous MI \\
(including left bundle branch block (LBBB)). \\
ACE, angiotensin converting enzyme; anti-huhsp60, anti-human heat \\
shock protein 60; anti-mhsp65, anti-mycobacterial heat shock protein \\
65 ; CRP, C reactive protein. \\
\end{tabular}

continuous covariates and the antibody titres, and twosample $t$ tests were used to compared mean titres of binary variates. The individual relation of each covariate to both the hard and the hard plus soft end points separately was assessed by univariate Cox proportional hazards models, with hazard ratios (and 95\% confidence intervals (CIs) with associated Wald p values) calculated for the stated increment in the covariate (for example, an increase of five years in age, or the presence or absence of diabetes). The quartiles of each antibody titre and a binary indicator of high ( $\geqslant 90$ th centile) antibody titre were also investigated. Kaplan-Meier survival plots stratified by the anti-huhsp60 titre quartiles were drawn. Multivariate Cox proportional hazards models were used to adjust any effect seen univariately for the antihuhsp60 titres (both as a continuous covariate and in quartiles) for age, history of hypertension, diabetes, smoking, and $\log (\mathrm{CRP})$. All analyses were performed with SAS 8.2 for Windows (SAS Institute, Cary, North Carolina, USA). No adjustment has been made for multiple testing.

\section{RESULTS}

\section{Patients}

A total of 710 consecutive patients were enrolled from a single centre. Of these, 33 were subsequently found to have had an MI (on the basis of development of new pathological Q Waves) at the time of index admission and were therefore excluded, leaving 677 patients. Also samples from 89 patients were lost, assumed to be missing at random, leaving a cohort of 588 patients with analysable data. Of these 588 patients, 13 did not have an anti-huhsp60 measurement and 10 did not have an anti-mhsp65 measurement. Table 1 summarises baseline clinical characteristics of the 588 patients.

\section{Follow up and clinical outcome}

Only one patient was lost to follow up after hospital discharge. During follow up (mean of 304 days, range 1788 days), 277 patients had any study outcome end point, of 
Table 2 Univariate hazard ratios for other risk factors (used later as adjusting covariates in the multivariate models)

\begin{tabular}{|c|c|c|c|c|}
\hline \multirow[b]{2}{*}{ Covariate } & \multicolumn{2}{|l|}{ Hard end point } & \multicolumn{2}{|c|}{ Hard plus soft end point } \\
\hline & $\mathrm{HR}(95 \% \mathrm{Cl})$ & p Value & $\mathrm{HR}(95 \% \mathrm{Cl})$ & p Value \\
\hline Age (5 year increase) & $1.25(1.12$ to 1.38$)$ & $<0.0001$ & $1.00(0.95$ to 1.05$)$ & 0.92 \\
\hline History of hypertension & $1.53(0.96$ to 2.44$)$ & 0.073 & $1.21(0.96$ to 1.53$)$ & 0.11 \\
\hline Diabetes & $1.91(1.11$ to 3.30$)$ & 0.020 & $1.11(0.81$ to 1.54$)$ & 0.52 \\
\hline Log (sensitive CRP) & $1.42(1.20$ to 1.68$)$ & $<0.0001$ & $1.05(0.97$ to 1.15$)$ & 0.24 \\
\hline Smoking & $0.76(0.46$ to 1.24$)$ & 0.27 & $0.97(0.83$ to 1.12$)$ & 0.64 \\
\hline
\end{tabular}

whom 7l had a hard end point first (CHD death or non-fatal MI) and 206 a soft end point initially (PTCA or CABG or angiography or readmission to hospital for chest pain). The numbers of patients experiencing each component of the composite hard plus soft outcome were as follows: 179 were readmitted for chest pain; 81 underwent angiography; 32 underwent PTCA; 32 underwent CABG; 54 died of CHD causes; 22 had a non-fatal MI.

\section{Antibody titres and baseline variables}

The Spearman rank correlation between anti-huhsp60 and anti-mhsp65 titre was 0.2 . Both antibodies were weakly positively correlated with age $(r=0.20)$ and with CRP $(r=0.15)$. There was no correlation with white cell count or with creatinine. There was no significant relation between either of the antibody titres and any of the categorical baseline variables in table 1 . In particular it should be noted that there was no association between antibody titres and history of atherosclerosis (either CHD or stroke). There were trends for patients taking long term aspirin to have lower titres of both anti-huhsp60 $(\mathrm{p}=0.07)$ and anti-mhsp65 $(\mathrm{p}=0.1)$.

Table 2 shows univariate hazard ratios (with 95\% CIs and $\mathrm{p}$ values) for other risk factors.

\section{Anti-huhsp60 titres and prognosis}

Table 3 details univariate and multivariate hazard ratios (with 95\% CIs and p values) for anti-huhsp60 titre. In univariate analysis there was a trend for patients with increased titres of anti-huhsp60 to have an increased risk of a hard end point (CHD death or non-fatal MI; hazard ratio $1.82,95 \%$ CI 0.92 to 3.62 comparing highest versus lowest

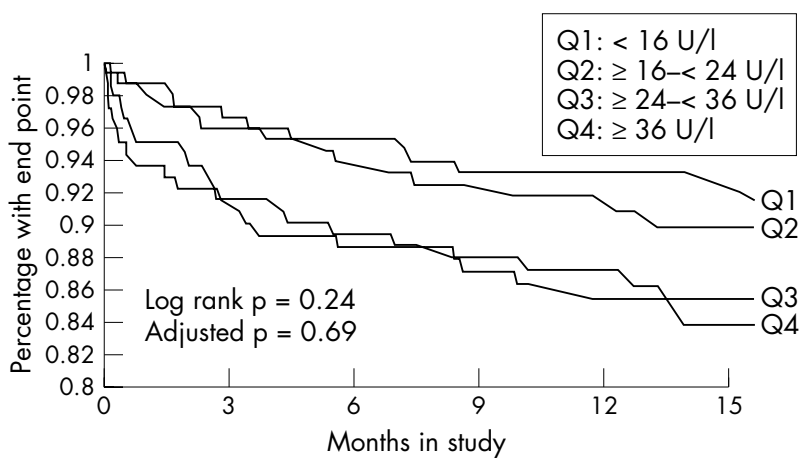

Figure 1 Kaplan-Meier survival plot of time to first hard end point by anti-human heat shock protein 60 (anti-huhsp60) quartiles (Q1 to Q4).

quartiles, $\mathrm{p}=0.087$ ). We found a graded increase in risk for the hard end point, with hazard ratios increased by about $80 \%$ in the top two quartiles, but overall these differences did not achieve significance.

Patients with raised concentrations of anti-huhsp60 did have a significantly increased risk for the composite hard and soft end point. This risk persisted after adjustment for covariates that have been previously shown to influence antihuhsp60 titre (age, hypertension, diabetes, and smoking) and the best studied inflammatory predictor, CRP. The hazard ratio was 1.56 (95\% CI 1.09 to 2.23) comparing highest versus lowest quartiles $(p=0.015)$. All patients above the first quartile $(\geqslant 16 \mathrm{U} / \mathrm{l})$ had an increased risk, with a hazard ratio about 50\% higher. Figures 1 and 2 shows Kaplan-Meier

Table 3 Univariate and multivariate (adjusted for covariates listed in table 2) hazard ratios for various characterisations of the anti-huhsp60 titre for the end points

\begin{tabular}{|c|c|c|c|c|}
\hline \multirow[b]{2}{*}{ Covariate } & \multicolumn{2}{|l|}{ Univariate } & \multicolumn{2}{|l|}{ Multivariate } \\
\hline & HR $(95 \% \mathrm{Cl})$ & p Value & HR $(95 \% \mathrm{Cl})$ & p Value \\
\hline \multicolumn{5}{|l|}{ Hard end point } \\
\hline Log (anti-huhsp60) & $1.30(0.98$ to 1.74$)$ & 0.071 & $1.08(0.80$ to 1.46$)$ & 0.61 \\
\hline$\geqslant 90$ th centile anti-huhsp 60 & 1.27 (0.63 to 2.57$)$ & 0.51 & $0.94(0.45$ to 1.94$)$ & 0.86 \\
\hline \multicolumn{5}{|l|}{ Anti-huhsp60 quartiles (U/I) } \\
\hline$<16$ & 1.00 & NA & 1.0 & NA \\
\hline$\geqslant 16-<24$ & $1.21(0.57$ to 2.53$)$ & 0.62 & $1.18(0.56$ to 2.48$)$ & 0.66 \\
\hline$\geqslant 24-<36$ & $1.76(0.87$ to 3.54$)$ & 0.11 & $1.52(0.75$ to 3.07$)$ & 0.25 \\
\hline$\geqslant 36$ & $1.82(0.92$ to 3.62$)$ & 0.087 & 1.35 (0.67 to 2.72$)$ & 0.41 \\
\hline Overall $p$ value & & 0.24 & & 0.69 \\
\hline \multicolumn{5}{|l|}{ Hard plus soft end point } \\
\hline Log (anti-huhsp60) & 1.09 (0.93 to 1.27$)$ & 0.29 & 1.09 (0.93 to 1.28 ) & 0.28 \\
\hline$\geqslant 90$ th centile anti-huhsp 60 & $0.94(0.63$ to 1.40$)$ & 0.77 & 0.91 (0.61 to 1.37$)$ & 0.66 \\
\hline \multicolumn{5}{|l|}{ Anti-huhsp60 quartiles (U/I) } \\
\hline$<16$ & 1.00 & NA & 1.0 & NA \\
\hline$\geqslant 16-<24$ & $1.61(1.14$ to 2.26$)$ & 0.0067 & 1.64 (1.16 to 2.32$)$ & 0.0051 \\
\hline$\geqslant 24-<36$ & 1.37 (0.96 to 1.95$)$ & 0.086 & 1.41 (0.98 to 2.03$)$ & 0.066 \\
\hline$\geqslant 36$ & 1.50 (1.06 to 2.13$)$ & 0.021 & 1.56 (1.09 to 2.23 ) & 0.015 \\
\hline Overall $p$ value & & 0.042 & & 0.031 \\
\hline
\end{tabular}




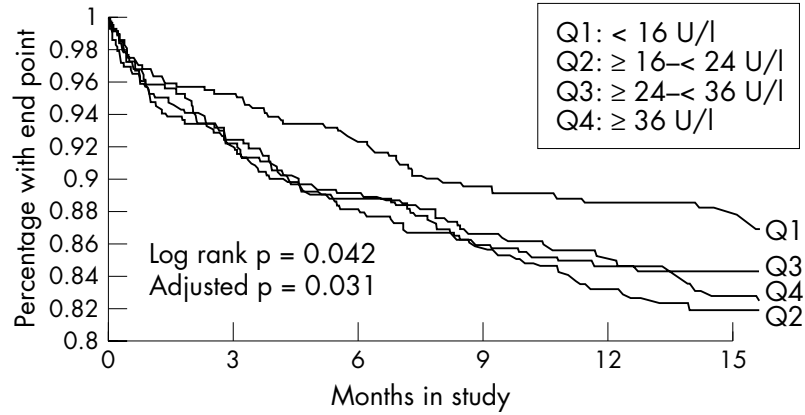

Figure 2 Kaplan-Meier survival plot of time to first hard plus soft end point by anti-huhsp60 quartiles (Q1 to Q4).

survival curves for anti-huhsp60 in quartiles for the hard end point and the combined hard plus soft end point.

\section{Anti-mhsp65 titres and prognosis}

Table 4 details the anti-mhsp65 data. There was no association between any of the characterisations of the anti-mhsp65 titre and either the hard end point or the composite end point.

\section{Anti-huhsp60 titre and prognosis in the troponin positive group}

Troponin $\mathrm{T}$ results from day $\mathrm{l}$ of the index admission were available for 526 patients, of whom 192 (37\%) were troponin positive $(>0.01 \mu \mathrm{g} / \mathrm{l})$. For the quartiles of anti-huhsp60 34\%, $30 \%, 39 \%$, and $43 \%$, respectively, were troponin positive. Refitting the same Cox models (as detailed above) to just the patients who were troponin positive showed no significant association between anti-huhsp60 and outcome (either hard or hard plus soft).

\section{DISCUSSION}

Our study indicates that patients admitted with acute cardiac chest pain and increased titres of anti-huhsp60 had an adverse one year prognosis for the hard plus soft outcome of CHD death or non-fatal MI plus angiography, PTCA, CABG, or readmission for cardiac chest pain. All patients above the first quartile $(\geqslant 16 \mathrm{U} / \mathrm{l})$ had an increased risk, with a hazard ratio about $50 \%$ higher. A more graded increase in risk was observed for the hard end point, with hazard ratios increased by about $80 \%$ in the top two quartiles. Overall, these differences did not achieve significance, in part due to the lower number of hard end points. These patterns were still in evidence after adjustment for the established cardiovascular risk factors of age, hypertension, diabetes, and smoking. Furthermore, the risk was independent of the best studied inflammatory predictor, CRP. Anti-mhsp65 titres were not predictive. Also there was no significant association between anti-huhsp60 and outcome in the troponin positive group. However, we suspect this is simply due to inadequate sample size, as there were only 120 combined end points in this group.

Evidence is accumulating that the inflammatory-immunological state of atherosclerosis is important in determining vulnerability to plaque rupture. In some cohorts, a higher concentration of CRP (a non-specific marker of inflammation) is a risk factor for progression of atherosclerosis to MI. ${ }^{18}$ Thus, it has been suggested ${ }^{19}$ that the biological state of a coronary lesion may be a more important determinant of the clinical outcome than, for example, the degree of stenosis. Interest has turned to other aspects of inflammation including antibodies to hsp60/65. The hsp60/65 family comprises huhsp60, mhsp65, the GroEL protein of Escherichia coli, huhsp60 of Chlamydia pneumoniae, hsp62 of Helicobacter pylori, and many others. ${ }^{20}$ Autoimmunity to huhsp60 provoked by infection with $C$ pneumoniae and perhaps other microorganisms has been postulated as one explanation for the observed associations between these infections and atherosclerosis. ${ }^{921-26}$

Four published prospective studies have examined the prognostic implications of anti-huhsp60 and anti-mhsp65. The first of these found that increased anti-mhsp 65 titres predicted mortality in a cohort with carotid atherosclerosis. ${ }^{15}$ The second found that anti-mhsp65 titres predicted progression of carotid atherosclerosis over five years. ${ }^{27}$ Neither study examined anti-huhsp60 titre.

The third study examined both anti-mhsp65 and antihuhsp60 titres in a nested case-control study of 386 patients with cardiovascular events (MI, cerebrovascular accident, cardiovascular death) and the same number of age and sex matched controls over a mean follow up of 4.5 years. ${ }^{14}$ Median serum concentrations of anti-mhsp65 antibodies was significantly higher in the patients than in the controls, and high titres ( $\geqslant 90$ th centile) of anti-mhsp65 antibodies

Table 4 Univariate and multivariate (adjusted for covariates listed in table 2) hazard ratios for various characterisations of the anti-mhsp65 titre for the end points

\begin{tabular}{|c|c|c|c|c|}
\hline \multirow[b]{2}{*}{ Covariate } & \multicolumn{2}{|l|}{ Univariate } & \multicolumn{2}{|l|}{ Multivariate } \\
\hline & HR $(95 \% \mathrm{Cl})$ & $\mathrm{p}$ Value & HR $(95 \% \mathrm{Cl})$ & p Value \\
\hline \multicolumn{5}{|l|}{ Hard end point } \\
\hline Log (anti-mhsp65) & 1.10 (0.86 to 1.40$)$ & 0.47 & 0.95 (0.74 to 1.23 ) & 0.70 \\
\hline$\geqslant 90$ th centile anti-mhsp65 & 1.31 (0.65 to 2.64 ) & 0.45 & 1.02 (0.50 to 2.07$)$ & 0.97 \\
\hline \multicolumn{5}{|l|}{ Anti-mhsp65 quartiles (U/I) } \\
\hline$<9<1<1$ & 1.0 & NA & 1.0 & NA \\
\hline$\geqslant 9-<17$ & 1.61 (0.81 to 3.22$)$ & 0.18 & $1.48(0.74$ to 2.99$)$ & 0.27 \\
\hline$\geqslant 17-<31$ & $1.52(0.75$ to 3.11$)$ & 0.25 & $1.23(0.60$ to 2.54$)$ & 0.57 \\
\hline$\geqslant 31$ & 1.36 (0.69 to 2.78 ) & 0.40 & 0.95 (0.46 to 1.97$)$ & 0.90 \\
\hline Overall $\mathrm{p}$ value & & 0.57 & & 0.52 \\
\hline \multicolumn{5}{|l|}{ Hard plus soft end point } \\
\hline Log (anti-mhsp65) & $0.97(0.86$ to 1.10$)$ & 0.63 & $0.97(0.86$ to 1.11$)$ & 0.68 \\
\hline$\geqslant 90$ th centile anti-mhsp 65 & 0.87 (0.58 to 1.32 ) & 0.52 & $0.87(0.57$ to 1.31$)$ & 0.50 \\
\hline \multicolumn{5}{|l|}{ Anti-mhsp65 quartiles (U/I) } \\
\hline$<9<0$ & 1.0 & NA & 1.0 & NA \\
\hline$\geqslant 9-<17$ & 1.06 (0.77 to 1.46$)$ & 0.74 & 1.03 (0.74 to 1.43 ) & 0.87 \\
\hline$\geqslant 17-<31$ & 0.76 (0.53 to 1.07 ) & 0.12 & $0.76(0.53$ to 1.08$)$ & 0.12 \\
\hline$\geqslant 31$ & 0.94 (0.67 to 1.30 ) & 0.69 & 0.94 (0.67 to 1.32$)$ & 0.74 \\
\hline Overall $p$ value & & 0.27 & & 0.33 \\
\hline
\end{tabular}


were found significantly more often among patients than among controls $(13.2 \% v 6.6 \%, \mathrm{p}=0.008) .{ }^{14}$ Median titres of anti-huhsp60 were similar in patients and controls.

The fourth study ${ }^{9}$ examined separately titres of IgA and IgG antibodies to huhsp60 in a nested case-control study. IgA but not IgG huhsp titres were a significant risk factor for coronary events (odds ratio 2.0, 5\% CI 1.1 to 3.6, when the fourth and first quartiles were compared). Further, they showed that the combination of an increased IgA antihuhsp60 with increased CRP and increased C pneumoniae IgA antibody titre conferred an odds ratio of 5.0 (95\% CI 1.8 to 14.2). ${ }^{9}$

There are several possible explanations for the contrast in results between our study and other prospective studies. Most important, the patient populations were quite different. Our cohort was unique in being recruited when they were unstable, when the atherosclerotic inflammatory state may be postulated to be quite different from the stable baseline state in the four other studies. There is also some evidence that acute ischaemia alters the expression of huhsp $60,{ }^{28}$ which is likely to influence anti-huhsp60 titre. Thus, our study is very importantly different from the other studies in this regard. There was one other minor difference between our cohort and that of Veres et $a l^{14}$ in the proportion of patients who had had a previous MI. In our group only 19.9\% had had a previous MI compared with $66.5 \%$ of patients in their study. ${ }^{14}$ Previous MI has been suggested to result in an important decrease in serum concentration of anti-huhsp60 antibodies. ${ }^{8}$ It seems likely, therefore, that titres of anti-hsp60 and the anti-hsp60-hsp60 reaction may have differing implications or effects in the three major stages of CHD (that is, chronic stable angina, unstable angina, and in the days and weeks after acute MI.) Stollberger and Finsterer noted the need for further study of this effect in the three stages in their editorial in The Lancet. ${ }^{29}$ Our study is the first to examine this in the unstable angina population.

We confirmed the findings of Veres et al $^{14}$ that anti-mhsp65 and anti-huhsp 60 titres are only weakly correlated. This is perhaps surprising because, like all hsp families, hsp60/65 is highly conserved between species-for example, mhsp65 is $75 \%$ homologous with huhsp $60 .{ }^{30}$ The weak correlation in titres suggests that anti-mhsp65 titres cannot be used as a surrogate for anti-huhsp60 titres and that future human studies should focus on the immune response to huhsp60. In our study there was only a minor correlation between CRP titre and huhsp60 titre. To our knowledge only Veres et al ${ }^{14}$ have performed a similar analysis and they found no correlation. Furthermore, in our study the relation between anti-huhsp60 titre and subsequent events was unchanged after correction for CRP. This all suggests that, although CRP and anti-huhsp60 titres are both inflammatory markers, they influence atherosclerotic risk through differing mechanisms.

There were interesting trends for patients taking long term aspirin to have lower titres of both anti-huhsp60 ( $p=0.07)$ and anti-mhsp65 ( $p=0.1)$. This has not previously been examined. There are similar data for CRP and aspirin. Low dose aspirin treatment reduces serum CRP concentrations ${ }^{31}$ in patients with but not in those without coronary artery disease. $^{32} 33$ In men, low dose aspirin appears to have its greatest protective effect against MI when serum CRP concentrations are relatively high. ${ }^{34}$ These observations raise the possibility that low dose aspirin may actually protect against MI by reducing inflammation.

In conclusion, patients admitted with acute cardiac chest pain and increased titres of anti-huhsp60 had an adverse one year prognosis independent of other cardiovascular risk factors (age, hypertension, diabetes, and smoking). Furthermore, the risk was independent of the best studied inflammatory predictor, CRP. Anti-mhsp65 titres were not predictive.

\section{ACKNOWLEDGEMENTS}

This research study was funded by a British Heart Foundation Junior Research Fellowship (LEV) and project grant (DHB). Thanks to C McNeil for technical help. Recombinant mhsp65 was a gift from Dr H D A van Emden, National Institute of Public Health and Environmental Protection, Bilthoven, the Netherlands.

\section{Authors' affiliations}

D H Birnie, L E Vickers, S M Cobbe, Department of Medical Cardiology, Royal Infirmary, Glasgow, UK

W S Hillis, Department of Medicine and Therapeutics, University of Glasgow, Glasgow, UK

J Norrie, Centre for Randomised Healthcare Trials (ChaRT), Health Services Research Unit, Aberdeen University, Aberdeen, UK

This research study was funded by a British Heart Foundation Junior Research Fellowship (LEV) and project grant. There are no conflicts of interest to declare.

The work was performed in the Department of Medical Cardiology, Royal Infirmary, Glasgow, Scotland and the Department of Medicine and Therapeutics, University of Glasgow, Scotland.

\section{REFERENCES}

1 Xu Q, Dietrich $\mathrm{H}$, Steiner $\mathrm{HJ}$, et al. Induction of arteriosclerosis in normocholesterolemic rabbits by immunization with heat shock protein 65 . Arterioscler Thromb 1992;12:789-99.

2 Xu Q, Willeit J, Marosi M, et al. Association of serum antibodies to heat-shock protein 65 with carotid atherosclerosis. Lancet 1993;341:255-9.

3 Xu Q, Luef $G$, Weimann S, et al. Staining of endothelial cells and macrophages in atherosclerotic lesions with human heat-shock proteinreactive antisera. Arterioscler Thromb 1993;13:1763-9.

4 Xu Q, Kleindienst R, Waitz W, et al. Increased expression of heat shock protein 65 coincides with a population of infiltrating T lymphocytes in atherosclerotic lesions of rabbits specifically responding to heat shock protein 65. J Clin Invest 1993:91:2693-702.

5 Xu Q, Schett G, Seitz CS, et al. Surface staining and cytotoxic activity of heatshock protein 60 antibody in stressed aortic endothelial cells. Circ Res 1994;75:1078-85.

6 Xu Q, Kleindienst R, Schett $G$, et al. Regression of arteriosclerotic lesions induced by immunization with heat shock protein 65 -containing material in normocholesterolemic, but not hypercholesterolemic, rabbits. Atherosclerosis 1996; 123:145-55.

7 Birnie DH, Holme ER, McKay IC, et al. Association between antibodies to heat shock protein 65 and coronary atherosclerosis: possible mechanism of action of Helicobacter pylori and other bacterial infections in increasing cardiovascular risk. Eur Heart J 1998; 19:387-94.

8 Hoppichler F, Lechleitner M, Traweger C, et al. Changes of serum antibodies to heat-shock protein 65 in coronary heart disease and acute myocardial infarction. Atherosclerosis 1996;126:333-8.

9 Huittinen $T$, Leinonen $M$, Tenkanen $L$, et al. Synergistic effect of persistent Chlamydia pneumoniae infection, autoimmunity, and inflammation on coronary risk. Circulation 2003; 107:2566-70.

10 Prohaszka Z, Duba J, Horvath L, et al. Comparative study on antibodies to human and bacterial $60 \mathrm{kDa}$ heat shock proteins in a large cohort of patients with coronary heart disease and healthy subjects. Eur J Clin Invest $2001 ; 31: 285-92$

11 Zhu J, Quyyumi AA, Rott D, et al. Antibodies to human heat-shock protein 60 are associated with the presence and severity of coronary artery disease: evidence for an autoimmune component of atherogenesis. Circulation 2001;103:1071-5

12 Huittinen T, Leinonen M, Tenkanen L, et al. Synergistic effect of persistent Chlamydia pneumoniae infection, autoimmunity, and inflammation on coronary risk. Circulation 2003;107:2566-70.

13 Kiechl S, Egger G, Mayr M, et al. Chronic infections and the risk of carotid atherosclerosis: prospective results from a large population study. Circulation 2001;103:1064-70.

14 Veres A, Fust G, Smieja M, et al. Relationship of anti-60 kDa heat shock protein and anti-cholesterol antibodies to cardiovascular events. Circulation 2002; 106:2775-80.

15 Xu Q, Kiechl S, Mayr M, et al. Association of serum antibodies to heat-shock protein 65 with carotid atherosclerosis: clinical significance determined in a follow-up study. Circulation 1999;100:1169-74.

16 Xu Q, Willeit J, Marosi M, et al. Association of serum antibodies to heat-shock protein 65 with carotid atherosclerosis. Lancet 1993;341:255-9.

17 Birnie D, McKay IC, Veitch J, et al. Antimycobacterial hsp65 and rheumatoid factor titres in a population of normal twins: evidence of genetic control of rheumatoid factor. Clin Exp Immunol 1995;101:393-7.

18 Ridker PM. Clinical application of C-reactive protein for cardiovascular disease detection and prevention. Circulation 2003;107:363-9.

19 Alexander RW. Inflammation and coronary artery disease. N Engl J Med 1994;331:468-9. 
20 Wick G, Schett $G$, Amberger A, et al. Is atherosclerosis an immunologically mediated disease? Immunol Today 1995; 16:27-33

21 Fong IW, Chiu B, Viira E, et al. Chlamydial heat-shock protein-60 antibody and correlation with Chlamydia pneumoniae in atherosclerotic plaques. $J$ Infect Dis 2002;186:1469-73.

22 Mahdi OS, Horne BD, Mullen K, et al. Serum immunoglobulin G antibodies to chlamydial heat shock protein 60 but not to human and bacterial homologs are associated with coronary artery disease. Circulation 2002; 106: 1659-63.

23 Burian K, Kis Z, Virok D, et al. Independent and joint effects of antibodies to human heat-shock protein 60 and Chlamydia pneumoniae infection in the development of coronary atherosclerosis. Circulation 2001;103:1503-8.

24 Hirono S, Dibrov E, Hurtado C, et al. Chlamydia pneumoniae stimulates proliferation of vascular smooth muscle cells through induction of endogenous heat shock protein 60. Circ Res 2003:93:710-6.

25 Huittinen $T$, Leinonen $M$, Tenkanen $L$, et al. Synergistic effect of persistent Chlamydia pneumoniae infection, autoimmunity, and inflammation on coronary risk. Circulation 2003;107:2566-70.

26 Fong IW, Chiu B, Viira E, et al. Chlamydial heat-shock protein-60 antibody and correlation with Chlamydia pneumoniae in atherosclerotic plaques. $J$ Infect Dis 2002;186:1469-73.
27 Kiechl S, Egger G, Mayr M, et al. Chronic infections and the risk of carotid atherosclerosis: prospective results from a large population study. Circulation 2001; 103:1064-70.

28 Gupta S, Knowlton AA. Cytosolic heat shock protein 60, hypoxia, and apoptosis. Circulation 2002;106:2727-33.

29 Stollberger C, Finsterer J. Atherosclerosis: infection-induced involvement of mitochondrial chaperonins [editorial]. Lancet 2003;362:1949-50.

30 Venner TJ, Gupta RS. Nucleotide sequence of mouse HSP60 (chaperonin, GroEL homolog) cDNA. Biochim Biophys Acta 1990;1087:336-8.

31 Ikonomidis I, Andreotti F, Economou E, et al. Increased proinflammatory cytokines in patients with chronic stable angina and their reduction by aspirin. Circulation 1999;100:793-8.

32 Feldman M, Jialal I, Devaraj S, et al. Effects of low-dose aspirin on serum C reactive protein and thromboxane B2 concentrations: a placebo-controlled study using a highly sensitive C-reactive protein assay. J Am Coll Cardiol $2001 ; 37: 2036-41$

33 Feng D, Tracy RP, Lipinska I, et al. Effect of short-term aspirin use on C reactive protein. J Thromb Thrombolysis 2000;9:37-41.

34 Ridker PM. Cushman M. Stampfer MJ, et al. Inflammation, aspirin, and the risk of cardiovascular disease in apparently healthy men. N Engl J Med 1997;336:973-9.

\section{IMAGES IN CARDIOLOGY}

\section{Fibrin thrombus causing myocardial infarction in a patient with patent coronary arteries}

A 47 year old woman, without coronary artery disease risk factors, first noted severe chest tightness as she was hurrying to catch the subway (underground). Acute inferior wall ST segment elevation myocardial infarction was diagnosed.

Emergent coronary angiography revealed one vessel disease. The right coronary artery (RCA), the infarct related artery, was occluded with thrombus containing lesions over the middle portion (panel A, arrow). An Export aspiration catheter was advanced into the RCA for thrombosuction. However, poor coronary flow was still noted due to ineffectiveness of removing large visible thrombi in the RCA. Balloon angioplasty was performed later without success, too. Since thrombosuction was considered to be able to relieve the thrombus burden rapidly, the guiding catheter was deeply advanced to the lesion (panel B) using the balloon catheter as an anchor. A large thrombus was aspirated. The aspirated thrombus, size $10 \times 5 \times 3 \mathrm{~mm}$, white (panel D) was found to match the filling defect on the angiography. The final angiography revealed an excellent result (panel $\mathrm{C}$ ) with a TIMI 3 flow. The histopathological examination showed that the aspirated thrombus was composed of fibrin

material and few blood cells (panel E). The patient's hospital course was smooth and she was discharged four days later.
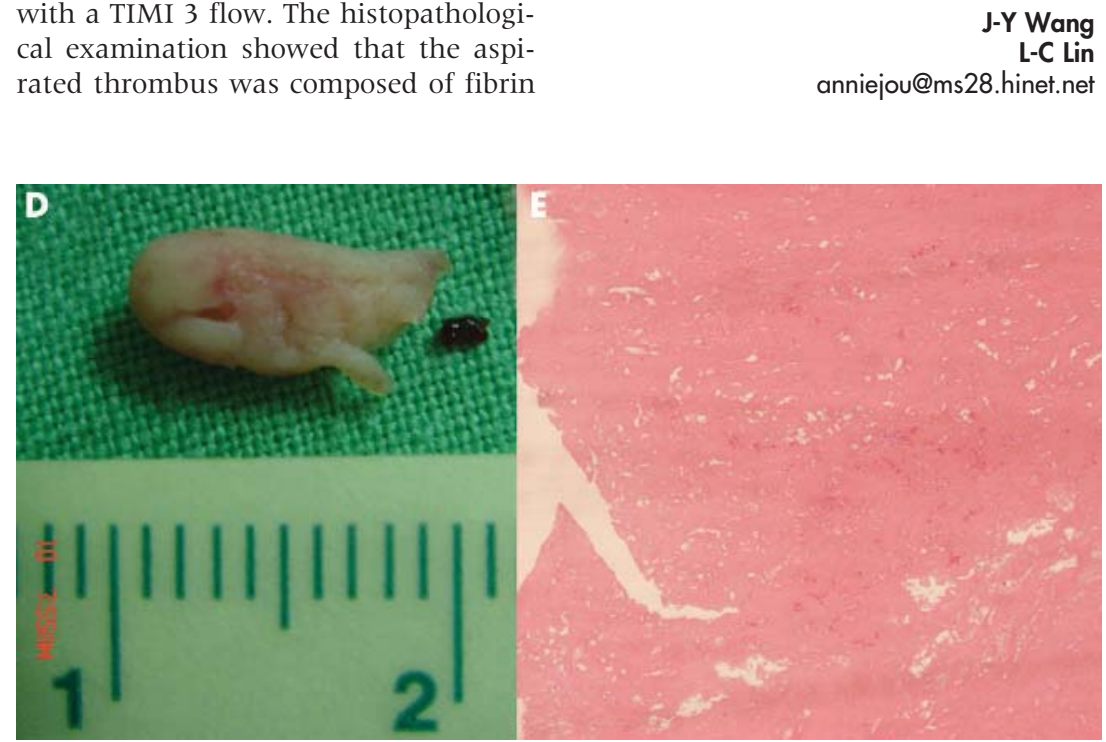
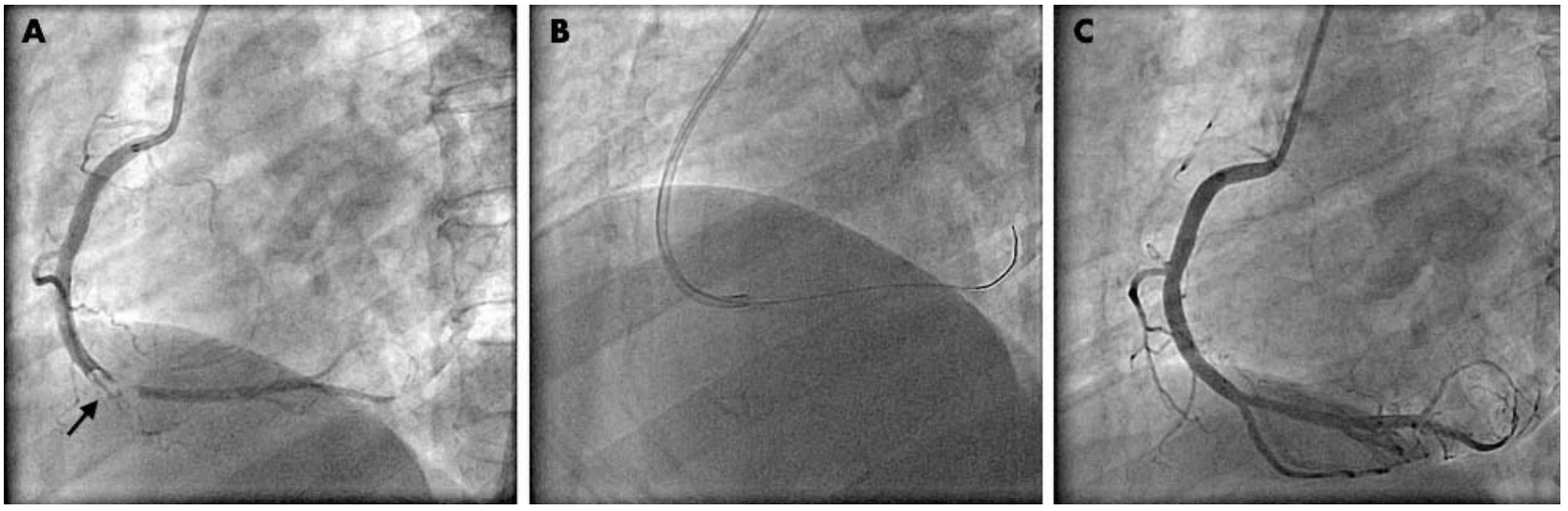